\title{
ON THE FOURIER EXPANSION OF STATIONARY RANDOM PROCESSES
}

\author{
R. C. DAVIS
}

The purpose of this note is to demonstrate that in the Fourier expansion of a quasistationary random continuous process with continuous covariance function, the amplitudes of the frequency components do not possess the desirable property of being mutually uncorrelated unless the process degenerates to a single random variable in its range of definition. Specifically we consider a real-valued continuous random process $x(t)$ observed during a time interval $T$. We assume that the mean and covariance function of $x(t)$ exist, so with no loss in generality we assume that

$$
E x(t) \equiv 0 \text { and } E x(s) x(t)=r(s-t),
$$

where $r(\tau)$ is an even function of $\tau$ and is continuous at $\tau=0$ with $r(0)=1$. It is then well known [1] that $r(\tau)$ is continuous for all $\tau$. Consider the Fourier expansion of $x(t)$ given by

$$
x(t)=a_{0}+\underset{m \rightarrow \infty}{\lim . \sum_{n=1}^{m}}\left(a_{n} \cos 2 \pi n \frac{t}{T}+b_{n} \sin 2 \pi n \frac{t}{T}\right)
$$

in which 1.i.m. denotes limit in the mean constructed from the covariance function $r(s-t)$. In the engineering literature [2] on random noise it is assumed quite frequently in the Fourier expansion of a quasistationary process that $E\left(a_{n} a_{m}\right)=0$ for $n \neq m$, and $E\left(a_{n} b_{m}\right)=0$ for all positive integers $n$ and $m$. What we show is that the only quasistationary process for which this can hold even for one pair of integers $n, m(n \neq m)$ and for all finite observation times ${ }^{1} T$ is the trivial process in which $r(s-t) \equiv 1$ for all $s$ and $t$.

It is well known that there exist quasistationary processes with discontinuous covariance functions for which the property holds that the amplitudes are mutually uncorrelated. The simplest example of one of these is a "pure white noise" which is characterized by $E \overline{x(t)^{2}}$ $=1 ; E x(s) x(t) \equiv 0$, for $s \neq t$. Moreover when the assumption of quasistationarity is dropped, and one considers processes for which the covariance function $r(s, t)$ may not be of the form $r(s-t)$, then there

Received by the editors December 10, 1952.

${ }^{1}$ It is easy to prove for sufficiently long observation times that as $T$ increases, the correlation coefficient between any two different amplitudes in the Fourier expansion decreases and approaches zero as $T$ approaches infinity. 
exist random processes with continuous covariance function for which the amplitudes of the Fourier components are mutually uncorrelated. The best known example is the Wiener process, for which $r(s, t)$ $=\sigma^{2} \min (s, t)$.

Even though we show that the above property does not exist for quasistationary processes with continuous covariance function $r(s-t)$ (except for the trivial process $x(t) \equiv a_{0}$, a single random variable in $T$ ), a solution to this problem is afforded by the orthogonal decomposition of a random continuous process obtained independently by several authors $[3 ; 4 ; 5]$. However, in order to obtain such a decomposition of a random process into uncorrelated components, one must solve a homogeneous linear integral equation, the kernel of which is the covariance function of the process with the range of integration being the time interval during which the process is observed. Although the eigenfunctions can be obtained explicitly in most practical cases, i.e., a quasistationary process with the spectral density a rational function of frequency, the eigenvalues must be obtained as solutions of a transcendental equation. In short the rigorous method of expansion of a random function into uncorrelated components is considerably more difficult to carry out than the simple task of expanding the function in a Fourier series.

H. B. Mann [6] has given a sufficient condition that a continuous random process with continuous covariance function can be expanded in a Fourier series which converges in the mean to the function $x(t)$. The condition is that $r(s, t)$ be a monotonoid and continuous function; i.e.,

$$
r(s, t)=h(s, t)-g(s, t)
$$

where $h$ and $g$ are two functions monotonic in $s$ and $t$ in the same sense. For our purposes we assume merely that the Fourier expansion converges in the mean in the interval $T$. It is convenient in the later manipulation to consider the expansion of $x(t)$ in exponentials. We assume then that for $0<t<T$,

$$
x(t)=\underset{m \rightarrow \infty}{\lim .} . \sum_{n=-m}^{n=+m} c_{n} \exp \left[2 \pi i n \frac{t}{T}\right]
$$

where

$$
c_{n}=\frac{1}{T} \int_{0}^{T} x(t) \exp \left[-2 \pi i n \frac{t}{T}\right] d t
$$

and 


$$
E\left(c_{n} \bar{c}_{m}\right)=\frac{1}{T^{2}} \int_{0}^{T} \int_{0}^{T} r(s-t) \exp \left[-2 \pi i \frac{(n s-m t)}{T}\right] d s d t .
$$

If we write

$$
a_{n}=c_{n}+c_{-n}, \quad b_{n}=i\left(c_{n}-c_{-n}\right),
$$

then

$$
x(t)=a_{0}+\underset{m \rightarrow \infty}{\lim .} \sum_{n=1}^{m}\left(a_{n} \cos 2 \pi n \frac{t}{T}+b_{n} \sin 2 \pi n \frac{t}{T}\right) .
$$

A. Khintchine has shown (loc. cit. p. 608) that the continuous covariance function of a continuous quasistationary random process admits the representation

$$
r(s-t)=\int_{-\infty}^{\infty} e^{i \omega(t-s)} d F(\omega)
$$

in which $F(\omega)$ is a bounded, nondecreasing function and is termed the spectral function of the process.

We state and prove the following theorem:

THEOREM. Given a real valued quasistationary random continuous process with spectral function $F(\omega)$ and variance $\int_{-\infty}^{\infty} d F(\omega)=1$, and for which the Fourier expansion in equation (2) converges in the mean. The necessary and sufficient condition that for any pair of unequal positive integers $n$ and $m$ and every finite time interval $T$ the amplitudes $a_{n}, a_{m}$, $b_{n}, b_{m}$ satisfy the relations $E a_{n} a_{m}=E a_{n} b_{m}=E a_{m} b_{n}=E b_{n} b_{m}=0$ is that $F(\omega)=0$ for $\omega<0$ and $F(\omega)=1$ for $\omega \geqq 0$.

Proof. Since it is more convenient to work with the expansion given in (1), we require the following lemma:

LEMMA. For any pair of unequal positive integers $n$ and $m$, the relations $E a_{n} a_{m}=E a_{n} b_{m}=E a_{m} b_{n}=E b_{n} b_{m}=0$ are equivalent to the relations $E c_{n} \bar{c}_{m}=E c_{n} \bar{c}_{-m}=0$.

The proof of the lemma is very simple and is obtained directly from the relations

$$
\begin{aligned}
& E c_{n} \bar{c}_{m}=4 E\left[\left(a_{n} a_{m}+b_{n} b_{m}\right)+i\left(a_{n} b_{m}-a_{m} b_{n}\right)\right], \\
& E c_{n} \bar{c}_{-m}=4 E\left[\left(a_{n} a_{m}-b_{n} b_{m}\right)+i\left(-a_{n} b_{m}-a_{m} b_{n}\right)\right] .
\end{aligned}
$$

Proceeding with the main proof,

$$
E c_{n} \bar{c}_{m}=\frac{1}{4 \pi^{2}} \int_{0}^{2 \pi} \int_{0}^{2 \pi} r[\alpha(u-v)] \exp [-i(n u-m v)] d u d v
$$


where $\alpha=T / 2 \pi$. By Khintchine's representation theorem, $r[\alpha(u-v)]$ $=\int_{-\infty}^{\infty} e^{\text {is } \alpha(\omega-\infty)} d F(\omega)$ where $F(\omega)$ is a bounded nondecreasing function. Inserting this value of $r[\alpha(u-v)]$ in the above, we obtain

$$
\begin{aligned}
E c_{n} \bar{c}_{m}=\frac{1}{4 \pi^{2}} \int_{0}^{2 \pi} \int_{0}^{2 \pi} \int_{-\infty}^{\infty} \exp [i(\alpha \omega-n) u \mid & \cdot \exp [-i(\alpha \omega-m) v] d F(\omega) d u d v .
\end{aligned}
$$

From the above lemma, the statement of the theorem is that the necessary and sufficient condition that the right-hand side of (3) should vanish for all positive $\alpha$ and any pair of integers $n \neq m$ is that $F(\omega)=0$ for $\omega<0$ and $F(\omega)=1$ for $\omega \geqq 0$. Since

$$
\begin{array}{r}
\frac{1}{4 \pi^{2}} \int_{0}^{2 \pi} \int_{0}^{2 \pi} \int_{-\infty}^{\infty}|\exp [i(\alpha \omega-n) u] \exp [-i(\alpha \omega-m) v]| \\
\cdot d F(\omega) d u d v \leqq r(0),
\end{array}
$$

we may invert the order of integration in (3) and obtain, after integration with respect to $u$ and $v$,

$$
E\left(c_{n} \bar{c}_{m}\right)=\frac{1}{\pi^{2}} \int_{-\infty}^{\infty} \frac{\sin ^{2} \pi \alpha \omega}{(\alpha \omega-n)(\alpha \omega-m)} d F(\omega) .
$$

The proof of the sufficiency of the theorem is obvious from (4). In order to prove the necessity, we denote the dependence of $E\left(c_{n} \bar{c}_{m}\right)$ on $\alpha$ by writing

$$
J_{n m}(\alpha)=\frac{1}{\pi^{2}} \int_{-\infty}^{\infty} \frac{\sin ^{2} \pi \alpha \omega}{(\alpha \omega-n)(\alpha \omega-m)} d F(\omega) .
$$

Since $J_{n m}(\alpha) \equiv 0$ for $\alpha>0$,

(5) $\int_{0}^{\infty} \frac{J_{n m}(\alpha)}{\alpha} d \alpha=\frac{1}{\pi^{2}} \int_{0}^{\infty} \frac{d \alpha}{\alpha} \int_{-\infty}^{\infty} \frac{\sin ^{2} \pi \alpha \omega}{(\alpha \omega-n)(\alpha \omega-m)} d F(\omega)=0$.

Since for all $\alpha>0$,

$$
\int_{0}^{\infty} d \alpha \int_{-\infty}^{\infty}\left|\frac{\sin ^{2} \pi \alpha \omega}{\alpha(\alpha \omega-n)(\alpha \omega-m)}\right| d F(\omega)<\infty,
$$

we may invert the order of integration in (5) and obtain

$$
\int_{-\infty}^{\infty} g_{n m}(\omega) d F(\omega)=0
$$

in which 


$$
\begin{aligned}
g_{n m}(\omega) & =\int_{0}^{\infty} \frac{\sin ^{2} \pi x d x}{x(x-n)(x-m)} & & (\text { for } \omega>0) \\
& =-\int_{-\infty}^{0} \frac{\sin ^{2} \pi x d x}{x(x-n)(x-m)} & & (\text { for } \omega<0), \\
& =0 & & (\text { for } \omega=0) .
\end{aligned}
$$

Now by using some of the usual tricks in evaluating infinite integrals (excluding the method of contour integration which appears to make the problem more complicated), it is not too difficult to show that

$$
\int_{-\infty}^{\infty} \frac{\sin ^{2} \pi x d x}{x(x-n)(x-m)}=0 .
$$

Moreover it can be shown that

$$
\left|\int_{0}^{\infty} \frac{\sin ^{2} \pi x d x}{x(x-n)(x-m)}\right|>0 .
$$

In view of this we see that the function $g_{n m}(\omega)$ is a constant unequal to zero except at $\omega=0$, and there $g_{n m}(0)=0$.

Hence we conclude from (6), since $F(\omega)$ is nondecreasing with $F(-\infty)=0$ and $\int_{-\infty}^{\infty} d F(\omega)=1$, that

$$
\begin{array}{ll}
F(\omega)=0, & \text { for } \omega<0, \\
F(\omega)=1, & \text { for } \omega \geqq 0,
\end{array}
$$

and the theorem is proved.

From Khintchine's representation theorem for $r(\tau)$ in terms of $F(\omega)$, we see that $r(\tau) \equiv 1$, for all $\tau$. By substituting this value in the expression for $E\left(c_{n} \bar{c}_{m}\right)$, we see that the above spectral function corresponds to the random process

$$
x(t) \equiv c_{0}=a_{0}
$$

with $a_{0}$ a random variable with

$$
E a_{0}=0 \text { and } E a_{0}^{2}=1 .
$$

I wish to acknowledge the benefit of several discussions with Professor R. P. Dilworth, California Institute of Technology.

\section{REFERENCES}

1. A. Khintchine, Korrelationstheorie der stationären stochastichen Prozesse, Math. Ann. vol. 109 (1934) pp. 604-615.

2. S. Goldman, Frequency analysis, modulation and noise, McGraw-Hill, 1948, pp. 325-330. 
3. M. Kac and A. J. F. Siegert, An explicit representation of a stationary Gaussian process, Ann. Math. Statist. vol. 18 (1947) p. 438.

4. Kari Karhunen, Über lineare Methoden in der Wahrscheinlichkeitsrechnung, Annales Academiae Scientiarum Fennicae, Helsinki, 1947.

5. M. Loève, Fonctions aleatoires de second ordre, from the book by P. Lévy entitled Processus stochastiques et mouvement Brownien, Gauthier-Villars, 1948.

6. H. B. Mann, Introduction to the theory of stochastic processes depending on a continuous parameter, National Bureau of Standards Report 1293, May, 1951, chap. 6.

U. S. Naval Ordnance Test Station, Pasadena

\section{AN EXPRESSION FOR $\vartheta_{1}(n z) / \vartheta_{1}(z)$}

W. N. BAILEY

1. Introduction. In a recent paper [1] I used the formula

$$
\begin{aligned}
& \Pi\left[\left(1+q^{n} / z\right)\left(1+q^{n-1} z\right)\left(1-q^{2 n-1} / z^{2}\right)\left(1-q^{2 n-1} z^{2}\right)\left(1-q^{n}\right)\right] \\
&= \prod\left[\left(1-q^{3 n-2} z^{3}\right)\left(1-q^{3 n-1} / z^{3}\right)\left(1-q^{3 n}\right)\right] \\
&+z \prod\left[\left(1-q^{3 n-1} z^{3}\right)\left(1-q^{3 n-2} / z^{3}\right)\left(1-q^{3 n}\right)\right]
\end{aligned}
$$

where, in the products, $n$ takes all values from 1 to $\infty$, to simplify certain identities of the Rogers-Ramanujan type. It has been shown by Sears [2] (and independently by Miss Slater) that (1.1) can be derived from the relation connecting three products of four sigma functions, or alternatively from the corresponding relation connecting theta functions. Now (1.1) can be written in the form

$$
\begin{aligned}
& \Pi \frac{\left(1-q^{n-1} z^{2}\right)\left(1-q^{n} / z^{2}\right)}{\left(1-q^{n-1} z\right)\left(1-q^{n} / z\right)} \\
& =\Pi \frac{\left(1-q^{3 n}\right)}{\left(1-q^{n}\right)} \times\left[\Pi\left(1-q^{3 n-1} / z^{3}\right)\left(1-q^{3 n-2} z^{3}\right)\right. \\
& \left.\quad+z \prod\left(1-q^{3 n-2} / z^{3}\right)\left(1-q^{3 n-1} z^{3}\right)\right]
\end{aligned}
$$

and if we write

$$
S(z ; p)=\Pi\left(1-p^{n-1} z\right)\left(1-p^{n} / z\right)
$$

this formula can be written as

$$
\frac{S\left(z^{2} ; q\right)}{S(z ; q)}=\Pi \frac{\left(1-q^{3 n}\right)}{\left(1-q^{n}\right)}\left[S\left(q z^{3} ; q^{3}\right)+z S\left(q^{2} z^{3} ; q^{3}\right) \mid .\right.
$$

Received by the editors December 15, 1952. 